\title{
k. mertebeden periyodik katsayılı $x(n+k)=A(n) x(n)$ sistemi için yeni Schur kararlıık parametresi ve Schur kararlılık parametreleri arasındaki ilişkiler
}

\author{
New Schur stability parameter for $k$-th order system with periodic coefficients \\ $x(n+k)=A(n) x(n)$ and relations between Schur stability parameters
}

\author{
Ahmet DUMAN*1,a, Gülnur ÇELIKK KIZILKAN ${ }^{1, b}$ \\ ${ }^{I}$ Necmettin Erbakan Üniversitesi, Fen Fakültesi, Matematik-Bilgisayar Bölümü, 42090, Konya
}

- Geliş tarihi / Received: 10.03 .2020

• Düzeltilerek geliş tarihi / Received in revised form: 23.03.2021

- Kabul tarihi / Accepted: 05.04.2021

Öz

Bu çalışmada $k$. mertebeden $x(n+k)=A(n) x(n)$ periyodik katsayılı lineer fark denklem sisteminin Schur kararlılığının kalitesini gösteren yeni $\bar{\omega}_{2}(A, T)$ parametresi tanımlandı. $\bar{\omega}_{1}(A, T)$ parametresi ile yeni $\bar{\omega}_{2}(A, T)$ parametresi arasındaki eşitsizlikler elde edildi. Elde edilen eşitsizlikler yardımıyla $k$. mertebeden periyodik katsayılı lineer fark denklemlerinin, $\bar{\omega}_{1}(A, T)$ parametresine bağlı olarak daha önce verilen sonuçlar $\bar{\omega}_{2}(A, T)$ parametresi için yeniden ifade edildi. Ayrıca elde edilen sonuçlar bir nümerik örnek ile desteklendi.

Anahtar kelimeler: Bozunum sistemleri, Fark denklemleri, Hassasiyet, Periyodik katsayılar, Schur kararlılık

\begin{abstract}
In this study, a new parameter $\bar{\omega}_{2}(A, T)$ which indicates the quality of Schur stability of $k$-th order linear difference equation system with periodic coefficients $x(n+k)=A(n) x(n)$ has been defined. Inequalities between parameter $\bar{\omega}_{1}(A, T)$ and new parameter $\bar{\omega}_{2}(A, T)$ have been obtained. With the help of obtained inequalities, the previously given results for $k$-th order linear difference equations with periodic coefficients which depends on the parameter $\bar{\omega}_{1}(A, T)$ have been reexpressed for $\bar{\omega}_{2}(A, T)$. In addition, the obtained results have been supported by a numerical example.
\end{abstract}

Keywords: Perturbation systems, Difference equations, Sensitivity, Periodic coefficients, Schur stability

\footnotetext{
${ }^{* a}$ Ahmet DUMAN; aduman @ erbakan.edu.tr, Tel: (0332) 32382 44, orcid.org/0000-0002-4022-5285

${ }^{\mathrm{b}}$ orcid.org/0000-0003-1538-082X
} 


\section{Giriş}

$A(n)=A(n+T) \in \mathbb{C}^{N \times N}$ periyodik matris ve $x(n) \in \mathbb{C}^{N \times 1}$ olmak üzere

$x(n+k)=A(n) x(n), x(s)=x_{s}(s=0,1,2, \ldots, k-1)$

$k$. mertebeden Cauchy problemini ele alalım. (1) sisteminin çözümü

$n=u k T+v, 0 \leq v \leq k T-1,0 \leq s \leq k-1$ ve $v=r k+s$ olmak üzere

$x(n)=X_{S}(r)\left(X_{S}(T)\right)^{u} x_{S}$

olarak elde edilmiştir (Çelik Kızılkan ve Duman, 2020). Bu çözüme dayanarak, $k$. mertebeden periyodik katsayılı (1) sisteminin Schur kararlılı̆g,$X=\left\{X_{S}(T) \mid s=0,1,2, \ldots, k-1\right\}$ matris ailesindeki her bir matrisin Schur kararlı olmasına denktir (Çelik Kızılkan ve Duman, 2020).

Benzer olarak Lyapunov teoremine göre "(1) sisteminin ( $X$ matris ailesindeki her bir matrisin) Schur kararl1 olmas1 için gerek ve yeter şart $s=0,1,2, \ldots, k-1$ için $X_{S}(T)$ matrisinin $X_{S}^{*}(T) F_{S} X_{S}(T)-F_{S}+I=0$ Lyapunov fark matris denkleminin (LFMD) $F_{S}=\sum_{j=0}^{\infty}\left(X_{S}^{*}(T)\right)^{j}\left(X_{S}(T)\right)^{j}=F_{s}^{*}>0$ tek çözümüne sahip olmasıdır" (Çelik Kızılkan ve Duman, 2020).

Çelik Kızılkan ve Duman (2020) tarafından (1) sisteminin Schur kararlılığının kalitesini gösteren kararlılık parametresi;

$\bar{\omega}_{1}(A, T)=\max _{0 \leq s \leq k-1}\left\|F_{s}\right\|$,

şeklinde tanıtılmıştır. Buna göre; $\bar{\omega}_{1}(A, T)<\infty$ oluyorsa sistem Schur kararlıdır. Aksi takdirde sistem Schur kararsızdır ve bu durumda $\bar{\omega}_{1}(A, T)=\infty$ olduğu kabul edilir.

\section{Ana sonuçlar}

\subsection{Semboller}

Çalışma için gerekli olan bazı gösterimler aşağıda tanıtılmıştır.

$$
\begin{aligned}
& \text { - } \quad \alpha=\max _{0 \leq j \leq k-1}\left\|\sum_{m=0}^{T-1} X_{j}^{*}(m) X_{j}(m)\right\| \text {, } \\
& \text { - } \beta_{S}=\max _{1 \leq i \leq T}\left\|Q_{S}(T, i)\right\|\left(1+(T-1) \max _{1 \leq i \leq T-1}\left\|Q_{S}(i, 0)\right\|\right) \\
& \text { - } \gamma_{s}=(T-1) \max _{1 \leq i \leq T}\left\|Q_{S}(T, i)\right\| \\
& \text { - } \mu_{s}=\max _{1 \leq i \leq T}\left\|Q_{s}(T, i)\right\| \times\left\{\begin{array}{cl}
\max _{0 \leq i \leq T-2}\|A(i k+s)\| ; & \max _{0 \leq i \leq T-2}\|A(i k+s)\| \leq 1 \\
\left(\max _{0 \leq i \leq T-2}\|A(i k+s)\|\right)^{T-2} ; & \max _{0 \leq i \leq T-2}\|A(i k+s)\|>1
\end{array}\right. \text {, } \\
& \text { - } Q_{s}(p, r)=\prod_{i=r}^{p-1} A(i k+s) \text {, } \\
& \text { - } \Psi_{s}(p, r)=\prod_{i=r}^{p-1} B(i k+s) \text {, } \\
& \text { - } \Delta_{1}^{S}=\sqrt{\left\|X_{S}(T)\right\|^{2}+\frac{\alpha}{\bar{\omega}_{2}(A, T)}}-\left\|X_{S}(T)\right\|, \\
& \text { - } \Delta_{2}^{S}=\max _{0 \leq i \leq T-1}\|B(i k+s)\|\left(\beta_{s}+\gamma_{s} \max _{1 \leq i \leq T-1}\left\|\Psi_{s}(i, 0)\right\|+\right. \\
& \left.\mu_{s} \sum_{r=2}^{T-1}\left[\sum_{l=1}^{r-1} \frac{r !}{l !(r-l) !}\left(\max _{0 \leq i \leq r-1}\|B(i k+s)\|\right)^{l}\right]\right), \\
& \text { - } \Delta_{3}^{S}=\frac{\max _{1 \leq i, j \leq T}\left\|Q_{S}(j, i)\right\|\left(1+(T-1) \max _{1 \leq i \leq T-1}\left\|X_{S}(i)\right\|\right)}{1-(T-1) \max _{1 \leq i, j \leq T}\left\|Q_{S}(j, i)\right\| \max _{1 \leq i \leq T-1}\|B(i k+s)\|} \max _{1 \leq i \leq T-1}\|B(i k+s)\|, \\
& -\Delta_{4}^{S}=\sqrt{\left\|X_{S}(T)\right\|^{2}+\frac{1}{\bar{\omega}_{2}(A, T)}}-\left\|X_{S}(T)\right\|, \\
& -\Delta_{2}^{S, *}=\sqrt{\left\|X_{S}(T)\right\|^{2}+\frac{\omega^{*}-\bar{\omega}_{2}(A, T)}{\omega^{*} \bar{\omega}_{2}(A, T)}}-\left\|X_{S}(T)\right\| .
\end{aligned}
$$




\subsection{Yeni Schur kararlılık parametresi}

$k$ mertebeden (1) sisteminin Schur kararlılı̆̆ının kalitesini gösteren ikinci bir kararlılık parametresini aşağıdaki şekilde tanımlayalım.

Tanım 1.1. $\left\{D_{s}\right\}_{s=0}^{k-1}=\left\{\sum_{m=0}^{T-1} X_{S}^{*}(m) X_{S}(m)\right\}_{s=0}^{k-1}$ olsun. $s=0,1,2, \ldots, k-1$ için $X_{S}^{*}(T) \Phi_{S} X_{S}(T)-\Phi_{s}+$ $D_{s}=0$ LFMD' nin çözümü $\Phi_{s}=\sum_{j=0}^{\infty} X_{S}^{*}(T)^{j} D_{s} X_{S}(T)^{j}=\sum_{j=0}^{\infty} X_{S}^{*}(j) X_{S}(j), \Phi_{s}=\Phi_{S}^{*}>0$ matris serisi olmak üzere; (1) sisteminin Schur kararlılığının kalitesini gösteren yeni kararlılık parametresi

$\bar{\omega}_{2}(A, T)=\max _{0 \leq s \leq k-1}\left\|\Phi_{s}\right\|$

şeklinde tanımlanır.

Buna göre (1) sistemi; $\bar{\omega}_{2}(A, T)<\infty$ ise Schur kararlı; aksi halde Schur kararsızdır ve $\bar{\omega}_{2}(A, T)=\infty$ şeklinde gösterilir.

Ayrıca $\omega^{*}>1$ pratik Schur kararlılık parametresi olmak üzere $\bar{\omega}_{i}(A, T) \leq \omega^{*}(i=1,2)$ eşitsizliği sağlanıyorsa (1) sistemine pratik Schur kararlı ya da kısaca $\omega^{*}-$ Schur kararlı, aksi halde $\omega^{*}-$ Schur kararsız sistem denir.

\section{3. $\bar{\omega}_{1}(A, T)$ ve $\bar{\omega}_{2}(A, T)$ kararlilık parametreleri arasındaki ilişkiler}

$\bar{\omega}_{1}(A, T)$ ve $\bar{\omega}_{2}(A, T)$ parametreleri arasındaki bazı eşitsizlikler aşağıda verilmiştir.

Teorem 2.1. $\bar{\omega}_{1}(A, T) \leq \bar{\omega}_{2}(A, T)$ ve $\bar{\omega}_{2}(A, T) \leq \alpha \bar{\omega}_{1}(A, T)$ eşitsizlikleri doğrudur.

İspat. $\left\{D_{S}\right\}_{s=0}^{k-1}=\left\{\sum_{m=0}^{T-1} X_{S}^{*}(m) X_{S}(m)\right\}_{s=0}^{k-1}$ olmak üzere

$$
\begin{aligned}
& \Phi_{s}=\sum_{j=0}^{\infty} X_{S}^{*}(T)^{j} D_{s} X_{S}(T)^{j}=\sum_{j=0}^{\infty} X_{S}^{*}(T)^{j}\left(\sum_{m=0}^{T-1} X_{S}^{*}(m) X_{S}(m)\right) X_{S}(T)^{j} \\
& =\sum_{j=0}^{\infty} X_{s}{ }^{*}(T)^{j}\left(I+X_{s}{ }^{*}(1) X_{S}(1)+\cdots+X_{s}^{*}(T-1) X_{S}(T-1)\right) X_{S}(T)^{j} \\
& =\sum_{j=0}^{\infty} X_{S}{ }^{*}(T)^{j} X_{S}(T)^{j}+\sum_{j=0}^{\infty} X_{S}{ }^{*}(T)^{j}\left(X_{S}{ }^{*}(1) X_{S}(1)+\cdots+X_{S}{ }^{*}(T-1) X_{S}(T-1)\right) X_{S}(T)^{j} \\
& =F_{S}+\sum_{j=0}^{\infty} X_{S}{ }^{*}(T)^{j}\left(X_{S}{ }^{*}(1) X_{S}(1)+\cdots+X_{S}{ }^{*}(T-1) X_{S}(T-1)\right) X_{S}(T)^{j}
\end{aligned}
$$

ve $s=0,1,2, \ldots, k-1$ için $\left\|\Phi_{s}\right\| \geq\left\|F_{s}\right\|$ olduğundan

$\bar{\omega}_{1}(A, T) \leq \bar{\omega}_{2}(A, T)$

olduğu görülür. Ayrıca;

$$
\begin{aligned}
\left\|\Phi_{s}\right\| & =\left\|\sum_{j=0}^{\infty} X_{S}^{*}(j) X_{S}(j)\right\|=\left\|\sum_{j=0}^{\infty} X_{S}^{*}(T)^{j}\left(\sum_{m=0}^{T-1} X_{S}^{*}(m) X_{S}(m)\right) X_{S}(T)^{j}\right\| \\
& \leq\left\|\sum_{m=0}^{T-1} X_{S}{ }^{*}(m) X_{S}(m)\right\|\left\|\sum_{j=0}^{\infty} X_{S}{ }^{*}(T)^{j} X_{S}(T)^{j}\right\|=\left\|\sum_{m=0}^{T-1} X_{S}{ }^{*}(m) X_{S}(m)\right\|\left\|F_{S}\right\|
\end{aligned}
$$

olduğundan

$\max _{0 \leq j \leq k-1}\left\|\Phi_{j}\right\| \leq \max _{0 \leq j \leq k-1}\left\|\sum_{m=0}^{T-1} X_{j}^{*}(m) X_{j}(m)\right\| \max _{0 \leq j \leq k-1}\left\|F_{j}\right\|$

elde edilir. $\alpha=\max _{0 \leq j \leq k-1}\left\|\sum_{m=0}^{T-1} X_{j}^{*}(m) X_{j}(m)\right\|$ olmak üzere

$\bar{\omega}_{2}(A, T) \leq \alpha \bar{\omega}_{1}(A, T)$

olduğu kolayca görülür.

Not 2.1. (2) den $\frac{1}{\bar{\omega}_{1}(A, T)} \leq \frac{\alpha}{\bar{\omega}_{2}(A, T)}$ olduğu açıktır.

Sonuç 2.1. Kararlılık parametreleri arasında $\frac{1}{\alpha} \bar{\omega}_{2}(A, T) \leq \bar{\omega}_{1}(A, T) \leq \bar{\omega}_{2}(A, T) \leq \alpha \bar{\omega}_{1}(A, T)$

veya buna denk olarak

$\frac{1}{\alpha \bar{\omega}_{1}(A, T)} \leq \frac{1}{\bar{\omega}_{2}(A, T)} \leq \frac{1}{\bar{\omega}_{1}(A, T)} \leq \frac{\alpha}{\bar{\omega}_{2}(A, T)}$

eşitsizlikleri sağlanır.

Teorem 2.2. (1) sistemi için

$\left|\bar{\omega}_{1}(A, T)-\bar{\omega}_{2}(A, T)\right| \leq(\alpha-1) \bar{\omega}_{1}(A, T)$

eşitsizliği doğrudur. 
İspat. Teorem 2.1. den $\bar{\omega}_{1}(A, T) \leq \bar{\omega}_{2}(A, T)$ ve $\bar{\omega}_{2}(A, T) \leq \alpha \bar{\omega}_{1}(A, T)$ olduğu bilindiğine göre, $\bar{\omega}_{2}(A, T)-\bar{\omega}_{1}(A, T) \leq \alpha \bar{\omega}_{1}(A, T)-\bar{\omega}_{1}(A, T)=(\alpha-1) \bar{\omega}_{1}(A, T)$

ve

$\bar{\omega}_{1}(A, T)-\alpha \bar{\omega}_{1}(A, T) \leq \bar{\omega}_{1}(A, T)-\bar{\omega}_{2}(A, T) \leq 0$

olur. Böylece

$\left|\bar{\omega}_{1}(A, T)-\bar{\omega}_{2}(A, T)\right| \leq(\alpha-1) \bar{\omega}_{1}(A, T)$

eşitsizliği sağlanır.

2.4. $k$. Mertebeden periyodik katsayıl $x(n+k)=A(n) x(n)$ lineer fark denklem sisteminin sürekliliği üzerine bazı sonuçlar

Şimdi $n \geq 0, T>0, A(n)=A(n+T)$ ve $B(n)=B(n+T)$ olmak üzere (1)' in

$y(n+k)=(A(n)+B(n)) y(n), y(s)=y_{s}(s=0,1,2, \ldots, k-1)$

bozunum sistemini ele alalım. (3) sisteminin fundamental matrisi $s=0,1,2, \ldots, k-1$ için $Y_{s}(n)=$ $\prod_{i=0}^{n-1}(A(i k+s)+B(i k+s)) \operatorname{dir}$.

(3) sisteminin aşikar çözümünün Schur kararlılığı için Çelik Kızılkan ve Duman (2020) tarafından $B(\mathrm{n})$ bozunum matrisi üzerine $\bar{\omega}_{1}(A, T)$ parametresine bağlı bazı şartlar verilmiştir. Şimdi bu sonuçları $\bar{\omega}_{2}(A, T)$ parametresine göre ifade edelim:

Teorem 2.3. $s=0,1,2, \ldots, k-1$ için (1) in monodromi matrisi $X_{S}(T)$ ve (3) ün monodromi matrisi $Y_{s}(T)$ olsun. Bu takdirde

$\left\|Y_{S}(T)-X_{S}(T)\right\|<\sqrt{\left\|X_{S}(T)\right\|^{2}+\frac{\alpha}{\bar{\omega}_{2}(A, T)}}-\left\|X_{S}(T)\right\|$

ifadesini sağlayan $B(n)$ bozunum matrisi için (3) sistemi Schur kararlıdır.

İspat. Bu teorem Çelik Kızılkan ve Duman'da (2020)'deki $\bar{\omega}_{1}(A, T)$ parametresi için verilen Teorem 3.1. in $\bar{\omega}_{2}(A, T)$ parametresine uyarlanmasıdır. Dolayısıyla, Teorem 3.1. in ispatında $\left\langle F_{S} Y_{S}((p+1) T) w, Y_{s}((p+\right.$ 1)T $w\rangle$ iç çarpımında $F_{S}$ matrisi yerine $\Phi_{s}$ matrisi alınıp ispat adım adım izlenirse (4) eşitsizliğine ulaşılır.

Fakat Teorem 2.3. deki sonucu elde etmek için Çelik Kızılkan ve Duman'daki (2020)'deki ispat tekniğini adım adım uygulamaya gerek yoktur. (4) eşitsizliğini elde etmek için Sonuç 2.1. de verilen $\frac{1}{\bar{\omega}_{1}(A, T)} \leq \frac{\alpha}{\bar{\omega}_{2}(A, T)}$ eşitsizliğini Çelik Kızılkan ve Duman (2020) Teorem 3.1. de kullanmak yeterlidir. Gerçekten,

Çelik Kızılkan ve Duman (2020), Teorem 3.1.' de $s=0,1,2, \ldots, k-1$ için (1) in monodromi matrisi $X_{S}(T)$ ve (3) ün monodromi matrisi $Y_{S}(T)$ olmak üzere

||$Y_{S}(T)-X_{S}(T)\left\|<\sqrt{\left\|X_{S}(T)\right\|^{2}+\frac{1}{\bar{\omega}_{1}(A, T)}}-\right\| X_{S}(T) \|$

ifadesini sağlayan $B(n)$ bozunum matrisi için (3) sistemi Schur kararlı olduğu ifade edilmiştir.

Sonuç 2.1. den $\frac{1}{\bar{\omega}_{1}(A, T)} \leq \frac{\alpha}{\bar{\omega}_{2}(A, T)}$ olduğu bilindiğine göre

||$Y_{S}(T)-X_{S}(T)\left\|<\sqrt{\left\|X_{S}(T)\right\|^{2}+\frac{\alpha}{\bar{\omega}_{2}(A, T)}}-\right\| X_{S}(T) \|$

olduğu açıktır. Böylece Teorem 2.3. ispatlanmış olur.

Teorem 2.4. (1) in Schur kararlı olması durumunda, $B(n)$ bozunum matrisi için

i) $\Delta_{1}^{2}<\Delta_{1}^{S}$

ii) ||$B(n) \|<\frac{\Delta_{1}^{s}}{\max _{1 \leq i, j \leq T}|| Q(j, i)||\left[\left(1+(T-1)\left(\max _{1 \leq i \leq T-1}|| X_{S}(i) \|+\Delta_{1}^{s}\right)\right]\right.}$

şartları sağlanırsa (3) bozunum sistemi Schur kararlıdır.

Teorem 2.5. (1) sistemi Schur kararlı olmak üzere, $B(n)$ bozunum matrisi için ||$B(n) \|<\frac{\Delta_{4}^{S}}{\max _{1 \leq i, j \leq T}|| Q(j, i) \|\left[\left(1+(T-1)\left(\max _{1 \leq i \leq T-1}|| X_{S}(i) \|+\Delta_{4}^{S}\right)\right]\right.}$ 
şartları sağlanırsa (3) bozunum sistemi Schur kararlıdır. Ayrıca;

$\bar{\omega}_{2}(A+B, T) \leq \frac{\bar{\omega}_{2}(A, T)}{1-\left(2|| X_{S}(T) \|+\Delta_{3}^{S}\right) \Delta_{3}^{S} \bar{\omega}_{2}(A, T)} ;\left\|\tilde{F}_{S}-F_{S}\right\| \leq \frac{\left(2-\left\|X_{S}(T)\right\|+\Delta_{3}^{S}\right) \Delta_{3}^{S} \bar{\omega}_{2}(A, T)^{2}}{1-\left(2-|| X_{S}(T) \|+\Delta_{3}^{S}\right) \Delta_{3}^{S} \bar{\omega}_{2}(A, T)}$ eşitsizlikleri doğrudur.

Teorem 2.6. $\omega^{*}-$ Schur kararlı (2) sistemi verilsin. Eğer $B(n)$ bozunum matrisi için

i) $\Delta_{2}^{S}<\Delta_{2}^{S, *}$,

ii) ||$B(n) \|<\frac{\Delta_{2}^{S, *}}{\max _{1 \leq j, r \leq T}|| Q(j, r) \|\left[\left(1+(T-1)\left(\max _{1 \leq r \leq T-1}|| X_{S}(r) \|+\Delta_{2}^{S, *}\right)\right]\right.}$

şartları sağlanırsa, (3) bozunum sistemi de $\omega^{*}$-Schur kararlı olur.

Not 2.2. $\bar{\omega}_{1}(A, T) \leq \bar{\omega}_{2}(A, T)$ ve $1 / \bar{\omega}_{1}(A, T) \leq \alpha / \bar{\omega}_{2}(A, T)$ eşitsizlikleri dikkate alınarak Teorem 2.4. ispat1 Duman ve Aydın' daki (2011) Teorem3-4 ve Teorem 2.4-2.5. in ispatı sırasıyla Duman vd.' daki (2016) Teorem 7-8 kaynaklarındaki teorem ispatlarına benzer şekilde kolaylıkla elde edilir. Tekrara düşmemek açısından ispatlar verilmemiştir.

Not 2.3. (1) sisteminde $k=1$ ve $T=1$ olması durumunda $\bar{\omega}_{2}(A, T)=\omega_{2}(A, T)=\omega(A)$ elde edilir. Bu durumda (1) sistemi 1. mertebeden sabit katsayılı sisteme dönüşür ve Teorem 2.3.-2.5. teoremleri 1 . mertebeden sabit katsayılı sistem için yazılan sonuçlar ile örtüşmektedir. $\mathrm{Bu}$ ise Teorem 2.3.-2.5. in literatürdeki benzer sonuçlarla olan uyumunu göstermektedir.

Not 2.4. Çalışmamızdaki hesaplamalar MVC (Bulgak ve Eminov, 2001) kullanılarak yapılmıştır.

Elde edilen eşitsizlikleri hesaplamak için aşağıdaki örneği ele alalım.

Örnek 2.1. $i=1,2,3$ için $A_{i}(n)$ matrisleri

$A_{1}(n)=\left(\begin{array}{cc}\frac{(-1)^{n}}{2} & 0 \\ 0 & \frac{1}{2}\end{array}\right), A_{2}(n)=\left(\begin{array}{cc}\frac{(-1)^{n}}{2} & 3 \\ 0 & 0.2\end{array}\right)$ ve $A_{3}(n)=\left(\begin{array}{cc}\sin \left(\frac{2 \pi}{3} n\right) & 0 \\ 0 & \cos \left(\frac{2 \pi}{3} n\right)\end{array}\right)$

olmak üzere 2. mertebeden periyodik katsayılı $x(n+2)=A(n) x(n)$ sistemini ele alalım. $A(n)$ katsayı matrisleri için elde ettiğimiz eşitsizliklerin sağlandığı Tablo 2.1. de görülmektedir.

Gösterimde kolaylık sağlaması açısından aşağıdaki eşitsizlikleri

$$
\begin{aligned}
& -\bar{\omega}_{2}(A, T) \leq \alpha \bar{\omega}_{1}(A, T) \\
& -\quad \frac{1}{\bar{\omega}_{1}(A, T)} \leq \frac{\alpha}{\bar{\omega}_{2}(A, T)} \\
& -\quad\left|\bar{\omega}_{1}(A, T)-\bar{\omega}_{2}(A, T)\right| \leq(\alpha-1) \bar{\omega}_{1}(A, T)
\end{aligned}
$$

şeklinde numaralandıralım.

Tablo 1. $A(n)$ Matrisleri için Hesaplanan Parametre Değerleri ve Eşitsizlikler

\begin{tabular}{llll}
\hline & $A_{1}(n)$ & $A_{2}(n)$ & $A_{3}(n)$ \\
\hline $\bar{\omega}_{1}(A, T)$ & 1.06667 & 8.09005 & 1.06667 \\
$\bar{\omega}_{2}(A, T)$ & 1.33333 & 24.1037 & 2.5 \\
$\alpha$ & 1.25 & 10.2889 & 2.5 \\
$(\mathrm{a})$ & $1.33333 \leq 1.3333375$ & $24.1037 \leq 83.237715$ & $2.5 \leq 2.666675$ \\
$(\mathrm{~b})$ & $0.937497 \leq 0.9375$ & $0.1236086 \leq 0.4268598$ & $0.937497 \leq 1$ \\
(c) & $0.266663 \leq 0.2666675$ & $16.01365 \leq 75.177665$ & $1.43333 \leq 1.600005$ \\
\hline
\end{tabular}

$\bar{\omega}_{1}(A, T), \bar{\omega}_{2}(A, T)$ parametrelerinden herhangi birine göre yazılmış bir sonucun diğer parametreye göre yazılmasını sağlayan (a), (b) ve (c) eşitsizlikleri ile ilgili nümerik örnekler Tablo 1. de verilmiştir. 


\section{Sonuçlar}

$\mathrm{Bu}$ çalışmada; $k$. mertebeden periyodik katsayılı (1) Cauchy problemi ele alınmıştır. (1) Cauchy problemi için elde edilen sonuçlar aşağıdaki gibi özetlenebilir.

- $\bar{\omega}_{2}(A, T)$ Schur kararl111k parametresi tanıtılmıştır.

- $\bar{\omega}_{1}(A, T)$ parametresi ile yeni $\bar{\omega}_{2}(A, T)$ parametresi arasındaki fonksiyonel eşitsizlikler elde edilmiştir.

Problemin Schur kararlı olması durumunda hangi bozunumlar altında sistemin Schur kararlı kaldığını gösteren $\bar{\omega}_{1}(A, T)$ parametresine bağlı olarak daha önce verilen süreklilik teoremleri, (1) problemi için yeni tanımlanan $\bar{\omega}_{2}(A, T)$ Schur kararlılık parametresine bağli olarak yeniden ifade edilmiştir.

\section{Kaynaklar}

Bulgak, H. and Eminov, D., 2001. Computer dialogue system MVC. Selcuk Journal Applied Mathematics, 2, 17-38.

Çelik Kızılkan, G. ve Duman, A., 2020. k. mertebeden periyodik katsayıl $\mathrm{x}(\mathrm{n}+\mathrm{k})=\mathrm{A}(\mathrm{n}) \mathrm{x}(\mathrm{n})$ lineer fark denklem sisteminin Schur kararlılığının hassasiyeti. Erzincan Üniversitesi Fen Bilimleri Enstitüsü Dergisi, 13 (3), 995-1003. http://dx.doi.org/ 10.18185/erzifbed.694163.

Duman, A. and Aydın, K., 2011. Sensitivity of Schur stability of monodromy matrix. Applied Mathematics and Computation, 217, 6663-6670. https://doi.org/10.1016/j.amc.2011.01.052

Duman, A., Çelik Kızılkan, G. and Aydın, K., 2016. Sensitivity of Schur stability of systems of linear difference equations with periodic coefficients. New Trends in Mathematical Sciences, 4 (2), 159-173.

http://dx.doi.org/10.20852/ntmsci.2016217826 